\title{
A Prepandemic Warning!
}

\author{
Robert G. Webster ${ }^{*}$
}

The goal of scientists is to provide knowledge that will benefit humankind. The scientific paper (1) published in the Proceedings of the National Academy of Sciences (online on June 29, 2020) has the potential of warning the global community of a future pandemic of disease. While the current focus on the coronavirus disease 2019 (COVID-19) pandemic caused by the COVID-19 virus is fully merited because of its catastrophic impact on public health and global economics, it is important not to forget that an influenza pandemic can be even more debilitating.

It has been just over 100 years since Mother Nature unleashed the devastating 1918 Spanish influenza virus that killed between 24.7 and 39.3 million persons worldwide. It is uncertain where this virus came from, but it was first reported on farms in the state of Kansas, USA and may well have come from swine. Each of the subsequent influenza pandemics of 1957, 1968, and 1977 had gene segments from the 1918 virus and the majority spread to or from swine. We do know that the H1N1 pandemic of 2009 (pdm/09 H1N1) was first detected in swine in Mexico with gene segments from Eurasian avian swine influenza viruses and the triple reassortant swine influenza virus from the Americas. The triple reassortant virus contained gene segments from the classical swine influenza virus (HA, NP, NS), human $\mathrm{H} 3$ influenza virus (PB1), and North American avian influenza viruses (PB2, PA). Each of these gene segments could be traced phylogenetically to avian influenza viruses from Eurasia or the Americas. Thus, the ultimate source of the pandemic influenza viruses of humans are the avian influenza viruses of the world and swine may serve as both the mixing vessel for the genesis of these viruses and the intermediate hosts in facilitating spread to humans.

The above paper establishes that another reassortant influenza virus containing the Eurasian avian swine hemagglutinin (HA) and neuraminidase (NA) with the majority of internal gene segments from the $\mathrm{pdm} / 09$ $\mathrm{H} 1 \mathrm{~N} 1$ virus became dominant in pigs in China in 2016. The following properties of this Eurasian avian reassortant influenza virus that are of concern for transmission to humans include:
1) Extensive diversity of the sequence of each of the gene segments is occurring, which indicates continued evolution.

2) Reassortment between the different swine influenza virus lineages has generated multiple different genotypes that all retain the Eurasian avian-like hemagglutinin and neuraminidase.

3) The dominant Eurasian avian-like reassortant preferentially bound to sialic acid $\alpha 2,6 \mathrm{Gal}$ receptors found in human cells.

4) These Eurasian avian-like reassortant viruses replicated to high titers in human airway epithelial cells.

5) The Eurasian avian-like reassortants caused severe disease signs in ferrets and transmitted efficiently by direct contact and by respiratory droplet spread to contact ferrets.

6) The reassortant Eurasian avian viruses were antigenically distinct from the pdm/09 H1N1 circulating in humans.

7) The current seasonal influenza vaccines to pdm/09 H1N1 do not provide protection against the Eurasian avian reassortant.

8) Serological surveillance of humans working in the swine industry showed that $10 \%$ had evidence of infection with the Eurasian avian reassortant influenza virus.

9) To date, there have been three reported cases of infection of humans with the Eurasian avian-like reassortant and one death.

10) The paper is extremely well illustrated with extensive referencing of earlier studies of influenza in swine in China.

The above findings indicate that the Eurasian avianlike reassortant influenza viruses have the ability to replicate in humans. Serological studies in humans indicate that pig to human transmission does occur. The key question is will these viruses acquire the ability to transmit human to human and initiate a pandemic? Integral to this question is understanding the factors that are currently limiting their spread. These Eurasian-avian viruses seem to tick many of the boxes we think necessary for a pandemic virus as detailed 
above; they bind human receptors, transmit in ferrets, and the human population seems mostly naïve to their HAs. What exactly is holding them back? Could it be that the virus is a mutation or two away from being optimally fit? Could it be that human immunity to the NA is important? Or could it just be good luck that these viruses haven't had more sustained human spread? Continued surveillance at the human-swine interface is clearly an important need. While we know some of the viral gene sequences associated with pathogenicity including PB2, PA-X, and NS we do not know the combination of gene segments and mutations that will allow predictions of human to human transmission.

Since China has the highest density of swine of any country in the world and also the continued circulation of all influenza virus lineages known to infect them, it would be extremely beneficial to continue surveillance at the human-swine interface (2-3). Pandemic preparedness resulting from surveillance at the humanswine interface would increase our knowledge of the molecular requirements for human to human transmission and facilitate early control and containment. Continued surveillance of humans working with swine is recommended by the authors of the paper and strongly supported by this author. It has the potential to provide new knowledge and benefit humankind globally.

Fundings: This work was funded by the National Institute of Allergy and Infectious Diseases (HHSN272201400006C) and American Lebanese Syrian Associated Charities (ALSAC).

doi: $10.46234 /$ ccdcw 2020.133

\# Corresponding author: Robert G. Webster, robert.webster@ stjude.org.

Submitted: June 29, 2020; Accepted: June 30, 2020

\section{REFERENCES}

1. Sun HL, Xiao YH, Liu JY, Wang DY, Li FT, Wang CX, et al. Prevalent Eurasian avian-like H1N1 swine influenza virus with 2009 pandemic viral genes facilitating human infection. P Natl Acad Sci USA 2020. http://dx.doi.org/10.1073/pnas.1921186117.

2. Song XH, Xiao H, Huang Y, Fu G, Jiang B, Kitamura Y, et al. Serological surveillance of influenza $A$ virus infection in swine populations in Fujian Province, China: no evidence of naturally occurring H5N1 infection in pigs. Zoonoses Public Hlth 2010;57(4): 291 - 8. http://dx.doi.org/10.1111/j.1863-2378.2009.01270.x.

3. Quan CS, Wang QL, Zhang J, Zhao M, Dai QG, Huang T, et al. Avian influenza A viruses among occupationally exposed populations, China, 2014-2016. Emerg Infect Dis 2019;25(12):2215 - 25. http://dx.doi.org/ 10.3201/eid2512.190261.

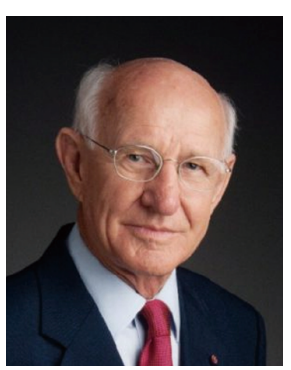

Robert G. Webster, PhD, DSc, FRS, NAS

Emeritus faculty, Division of Virology, Department of Infectious Diseases

St. Jude Children's Research Hospital 